\title{
INVERSE CORRELATION BETWEEN VEGF AND SOLUBLE VEGF RECEPTOR 2 IN POEMS WITH AIDP RESPONSIVE TO INTRAVENOUS IMMUNOGLOBULIN
}

\author{
CHIARA TERRACCIANO, MD, ${ }^{1}$ STEFANIA FIORE, MD, ${ }^{1}$ ELENA DOLDO, PhD, ${ }^{2}$ VITTORIO MANZARI, MD, ${ }^{2}$ \\ GIROLAMA ALESSANDRA MARFIA, MD, ${ }^{1}$ GIORGIO BERNARDI, MD, ${ }^{1}$ ROBERTO MASSA, MD, ${ }^{1}$ \\ and LOREDANA ALBONICI, PhD ${ }^{2}$ \\ ${ }^{1}$ Department of Neurosciences, Tor Vergata University of Rome and IRCCS Fondazione Santa Lucia, Via Montpellier 1, \\ I-00133 Rome, Italy \\ ${ }^{2}$ Department of Experimental Medicine, Tor Vergata University of Rome, Rome, Italy \\ Accepted 4 March 2010
}

ABSTRACT: POEMS (polyneuropathy, organomegaly, endocrinopathy, M-band, and skin changes) syndrome is characterized by chronic progressive polyneuropathy and plasma-cell dyscrasia. A major diagnostic criterion of POEMS is elevation of circulating vascular endothelial growth factor (VEGF), which is believed to play a pathogenic role in this disease. We report a case of POEMS that presented as relapsing acute inflammatory demyelinating polyneuropathy, in which complete remission after intravenous immunoglobulin (IVIg) treatment was unexpectedly observed. At clinical nadir, the VEGF level was 30 -fold higher, and the soluble form of VEGF receptor 2 (sVEGFR2), which acts as a decoy for VEGF, was 2.7-fold lower than normal. These changes combined might contribute to the pathogenesis of POEMS, inducing vascular permeability and tissue edema. At 9-month follow-up, during clinical remission, VEGF and SVEGFR2 were near normal values. sVEGFR2 reduction is a new finding in POEMS. IVIg treatment may benefit POEMS patients with acute neuropathy by downgrading VEGF release induced by inflammatory cytokines.

Muscle Nerve 42:445-448, 2010

Poems is a rare syndrome characterized by a progressive polyneuropathy associated with an underlying plasma-cell dyscrasia. The acronym POEMS (polyneuropathy, organomegaly, endocrinopathy, M-band, and skin changes) signifies several features of the syndrome. Polyneuropathy and monoclonal plasma cell disorder are present in all patients; to make the diagnosis of POEMS at least another major criterion (sclerotic bone lesions or VEGF elevation) and one minor criterion (organomegaly, endocrinopathy, extravascular volume overload, skin changes, papilledema, thrombocytosis) are required. ${ }^{1-3}$

Vascular endothelial growth factor (VEGF), the most potent angiogenic factor, is known to also have direct trophic effects on neuronal cells in

Abbreviations: AIDP, acute inflammatory demyelinating polyneuropathy; $\mathrm{CIDP}$, chronic inflammatory demyelinating polyneuropathy; CMAP, compound muscle action potential; CSF, cerebrospinal fluid; CT, computed tomography; EMG, electromyography; GBS, Guillain-Barré syndrome; IL-1, interleukin-1; IVIg, intravenous immunoglobulin; MUP, motor unit potential; $\mathrm{PE}$, plasma exchange; POEMS, polyneuropathy, organomegaly, endocrinopathy, M-band and skin changes; sVEGFR1, soluble VEGF receptor 1; TNF- $\alpha$, tumor necrosis factor- $\alpha$; VEGF, vascular endothelial growth factor; sVEGFR2, soluble VEGF receptor 2

Key words: acute polyneuropathy; IVIg; POEMS, sVEGFR2; VEGF

Correspondence to: R. Massa; e-mail: massa@uniroma2.it

(C) 2010 Wiley Periodicals, Inc.

Published online 29 June 2010 in Wiley InterScience (www.interscience. wiley.com). DOI 10.1002/mus.21718

VEGF and sVEGFR2 in POEMS Responsive to IVIg vivo and in vitro. ${ }^{4}$ The biological effects of VEGF are mediated by two main receptors, VEGFR1 and VEGFR2. VEGFR2 is the best characterized signaling receptor. It drives angiogenesis in health and disease and stimulates endothelial-cell proliferation, migration, navigation of tip cells, survival, and vascular permeability. ${ }^{5}$ Recently, a soluble form of VEGFR2 (sVEGFR2) has been discovered in human and mouse plasma, and its levels correlate inversely with tumor progression. ${ }^{6}$

Further, although sVEGFR2 can trap VEGF, it cannot displace VEGF from endothelial cells that express both receptors. In contrast, soluble VEGFR1 (sVEGFR1) can almost completely block VEGF-induced cell proliferation and migration due to its higher affinity for VEGF than VEGFR2. ${ }^{5}$

The typical peripheral neuropathy of POEMS is similar to chronic inflammatory demyelinating polyneuropathy (CIDP) and is believed to be caused by blood-nerve barrier dysfunction mediated by an increase of circulating VEGF. However, in contrast to the classic form of CIDP, it is not responsive to plasma exchange (PE) and intravenous immunoglobulin (IVIg) treatment., ${ }^{2,3}$

\section{CASE REPORT}

We report the case of a 57-year-old man with a 1year history of thrombocytosis who presented in September 2008 with acute onset of tingling and numbness of the hands. The symptoms subsequently involved the lower limbs with a distal-proximal progression, and they were associated with generalized asthenia and walking impairment. Neurological examination revealed sensory ataxia, decreased vibration and deep touch sensation in the lower limbs, moderate strength reduction in the distal portions of all four limbs, and absence of all deep tendon reflexes. Disability was grade 3 according to the Hughes GBS disability score ${ }^{7}$ (Fig. 1). Cerebrospinal fluid (CSF) showed no cells and an increased protein concentration $(65 \mathrm{mg} / \mathrm{dl})$.

The electrophysiological evaluation demonstrated widespread slowing of motor and sensory 


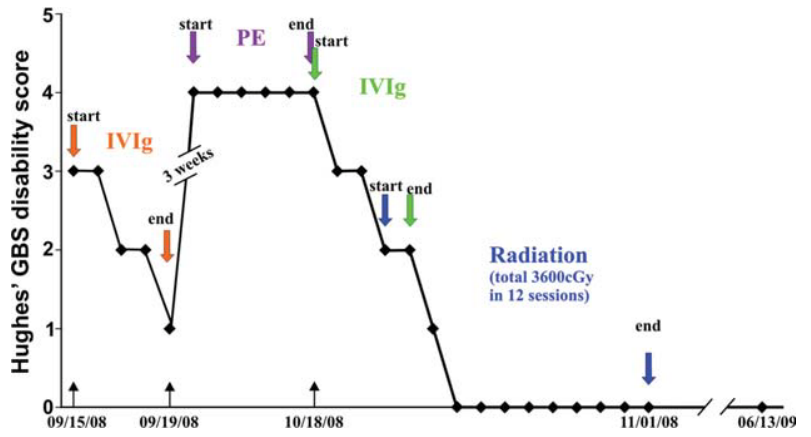

FIGURE 1. Clinical course in relation to therapies. PE, plasma exchange; IVIg, intravenous immunoglobulin; GBS, GuillainBarré syndrome. [Color figure can be viewed in the online issue, which is available at www.interscience.wiley.com.]

conduction velocities with prolonged distal latencies of compound muscle action potentials (CMAPs) in the lower limbs and increased F-wave latencies in all four limbs (Table 1). Needle electromyographic (EMG) examination of distal limb muscles showed impaired recruitment of motor unit potentials (MUPs) in the absence of spontaneous activity. Such findings, together with the acute onset and rapid progression (nadir in 10 days) of neurological symptoms, were suggestive of acute inflammatory demyelinating neuropathy (AIDP).

Blood tests were normal except for an increased platelet count $\left(936 \times 10^{3} / \mathrm{mm}^{3}\right)$. The patient was treated with IVIg for 5 days $(400 \mathrm{mg} /$

\begin{tabular}{|c|c|c|c|}
\hline & $\begin{array}{l}\text { Sept. } \\
2008\end{array}$ & $\begin{array}{l}\text { Oct. } \\
2008\end{array}$ & $\begin{array}{l}\text { June } \\
2009\end{array}$ \\
\hline \multicolumn{4}{|l|}{ Sensory nerves } \\
\hline \multicolumn{4}{|l|}{ Right radial } \\
\hline SNAP amplitude $(\mu \mathrm{V})$ & 23.0 & 17.0 & 14.0 \\
\hline SNCV (m/s) & 58.7 & 46.0 & 50.0 \\
\hline \multicolumn{4}{|l|}{ Right ulnar } \\
\hline SNAP amplitude $(\mu \mathrm{V})$ & 18.5 & 21.0 & 20.0 \\
\hline SNCV $(\mathrm{m} / \mathrm{s})$ & 48.0 & 45.8 & 51.2 \\
\hline \multicolumn{4}{|l|}{ Right sural } \\
\hline SNAP amplitude $(\mu \mathrm{V})$ & 9.4 & 7.1 & 8.3 \\
\hline $\mathrm{SNCV}(\mathrm{m} / \mathrm{s})$ & 36.1 & 33.3 & 48.3 \\
\hline \multicolumn{4}{|l|}{ Motor nerves } \\
\hline \multicolumn{4}{|l|}{ Right ulnar } \\
\hline Distal latency (ms) & 3.4 & 3.7 & 3.0 \\
\hline CMAP amplitude (mV) & 7.7 & 8.6 & 10.0 \\
\hline $\operatorname{MNCV}(\mathrm{m} / \mathrm{s})$ & 52.6 & 47.6 & 61.0 \\
\hline F-wave min latency (ms) & 36.2 & 37.0 & 32.5 \\
\hline \multicolumn{4}{|l|}{ Right tibial } \\
\hline Distal latency (ms) & 6.7 & 7.8 & 3.7 \\
\hline CMAP amplitude (mV) & 4.7 & 4.8 & 5.4 \\
\hline $\operatorname{MNCV}(\mathrm{m} / \mathrm{s})$ & 29.4 & 25.8 & 34.0 \\
\hline F-wave min latency (ms) & 80.7 & 84.7 & 68.5 \\
\hline \multicolumn{4}{|l|}{ Right peroneal } \\
\hline Distal latency (ms) & 7.6 & 7.8 & 4.8 \\
\hline CMAP amplitude (mV) & 6.4 & 4.7 & 4.3 \\
\hline $\operatorname{MNCV}(\mathrm{m} / \mathrm{s})$ & 31.2 & 28.5 & 34.1 \\
\hline
\end{tabular}

CMAP, compound motor action potential; MNCV, motor nerve conduction velocity; SNAP, sensory nerve action potential; SNCV, sensory nerve conduction velocity. $\mathrm{kg} /$ day), with substantial improvement of symptoms after the third day of infusion (from grade 3 to grade 1 for GBS disability score) (Fig. 1). After being almost asymptomatic for 3 weeks, the patient was re-admitted to hospital in October 2008 for a relapse of the neuropathic symptoms that occurred over 2 days. At this time, he had severe weakness of all limbs, dysphagia, and bilateral facial paresis (grade 4 for the GBS disability score) (Fig. 1). The CSF protein level was increased $(150 \mathrm{mg} / \mathrm{dl})$, and cells were not present. Nerve conduction studies showed pronounced signs of demyelination in all four limbs (Table 1), whereas EMG documented fibrillation potentials and positive sharp waves ( 6 or 7 per 10 needle positions) in all muscles.

Due to his rapidly deteriorating clinical condition, the patient underwent five PE sessions, without any improvement in neuropathy score (Fig. 1). Because the patient reported a significant weight loss (10 kg in 2 months), a total body computed tomography (CT) scan was performed to screen for an underlying neoplasm. There was a mixed sclerotic and lytic lesion of the pelvic bone (Fig. 2), which, on biopsy, was diagnosed as a lambda light-chain plasmacytoma. Bone marrow biopsy showed the presence of about 5\% plasma cells. Hormonal screening showed multiple endocrinopathy: testosterone $1.77 \mathrm{ng} / \mathrm{ml}$ (normal 2.88.0 ), parathyroid hormone $5.40 \mathrm{pg} / \mathrm{ml}$ (normal $14.0-72.0)$, adrenocorticotrophic hormone $1.0 \mathrm{pg} /$ $\mathrm{ml}$ (normal 5.0-60.0), and cortisol $2.7 \mu \mathrm{g} / \mathrm{dl}$ (normal 4.0-22.0). The association of a monoclonal plasmaproliferative disease with demyelinating peripheral neuropathy, sclerotic bone lesion, hormonal alterations, and thrombocytosis suggested the diagnosis of POEMS. ${ }^{2,3}$ An ophthalmologic examination which revealed bilateral papilledema further supported this diagnosis. At this point the patient underwent a new cycle of IVIg; treatment was again successful in leading to complete recovery

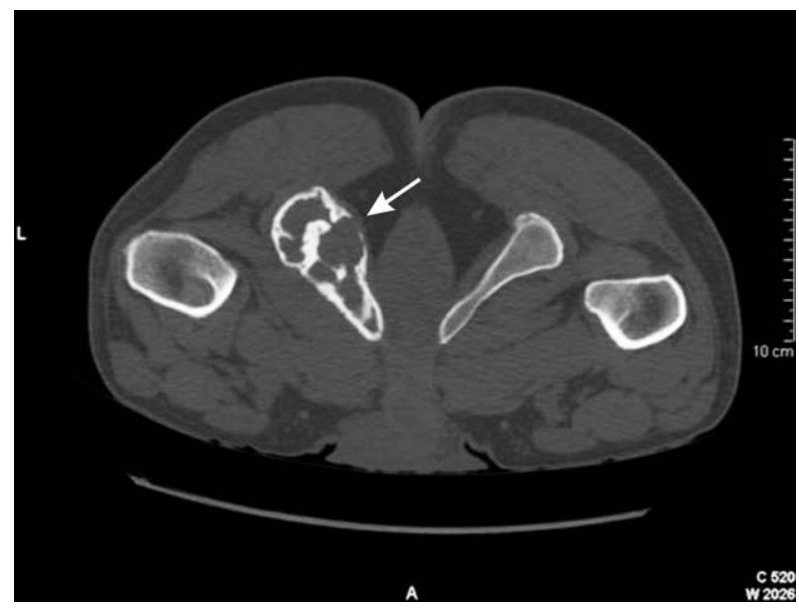

FIGURE 2. Total body CT scan. Plasmacytoma lesion with typical 'soap bubble' appearance in the pelvic bone.

MUSCLE \& NERVE 
of the neuropathy (from grade 4 to grade 0 for GBS disability score) within 7 days (Fig. 1). To treat the bone lesion and stabilize the remission, the patient received 12 applications of radiation therapy (total dose 3600 cGy) directed to the plasmacytoma.

After 9 months of follow-up the patient was still asymptomatic, and neurophysiological examination showed substantial improvement of nerve conduction values in the upper limbs; in the lower limbs, motor distal latencies and sensory conduction parameters returned to normal values, while motor conduction velocities improved slightly (Table 1). EMG showed polyphasic MUPs with increased amplitude and duration, in the presence of sporadic fibrillation potentials ( 1 or 2 per 10 needle positions).

\section{METHODS}

During the second hospitalization, we were able to perform serum assays of VEGF, sVEGFR1, and sVEGFR2 by enzyme immunoassay (Quantikine R\&D System), according to the manufacturer's instructions. Each kit was designed to eliminate mutual interference by ligands or other proteins in biological samples. The results were from three independent assays, each performed in duplicate. Because of the high concentration of VEGF, serum from the patient was analyzed either undiluted or after 1:5, 1:10, or 1:50 dilutions. Although the collection of sera could cause the activation of platelets and ensuing VEGF release, we measured serum concentration, because most studies were performed on serum rather than on plasma samples. ${ }^{8}$ Sera from 3 healthy age-matched volunteers were used as normal controls.

\section{RESULTS}

The VEGF serum level (mean 9272 pg/ml; range 6362-12,182) was about 30-fold higher than the mean value of 3 normal control subjects ${ }^{9}$ (279.4 $\mathrm{pg} / \mathrm{ml}$; range 189-386 pg/ml) (Fig. 3A). Remarkably, the sVEGFR2 serum level $(4485 \mathrm{pg} / \mathrm{ml}$; range 2273-5570 pg/ml) was 2.7-fold lower than the mean value of the 3 normal controls (12,031 pg/ $\mathrm{ml}$; range 10,410-12,895 pg/ml) (Fig. 3B), whereas the sVEGFR1 value (131 $\mathrm{pg} / \mathrm{ml}$; range 69.7-192.3 $\mathrm{pg} / \mathrm{ml}$ ) did not show significant changes compared with the mean value of the 3 normal controls (189.7 pg/ml; range $118.6-312.8 \mathrm{pg} / \mathrm{ml}$ ). At the 9month follow-up (June 2009), when the patient was in neurological remission, blood tests indicated an absence of monoclonal protein and a platelet count of $450 \times 10^{3} / \mathrm{mm}^{3}$. VEGF was decreased 6-fold compared with the first assay (1568 pg/ml; range $1376-1687 \mathrm{pg} / \mathrm{ml}$ ) and sVEGFR2 was increased (7378 pg/ml; range 5328$9965 \mathrm{pg} / \mathrm{ml}$ ) (Fig. 3). There was a slight decrease in sVEGFR1 levels (125.3 pg/ml; range 121-129.6
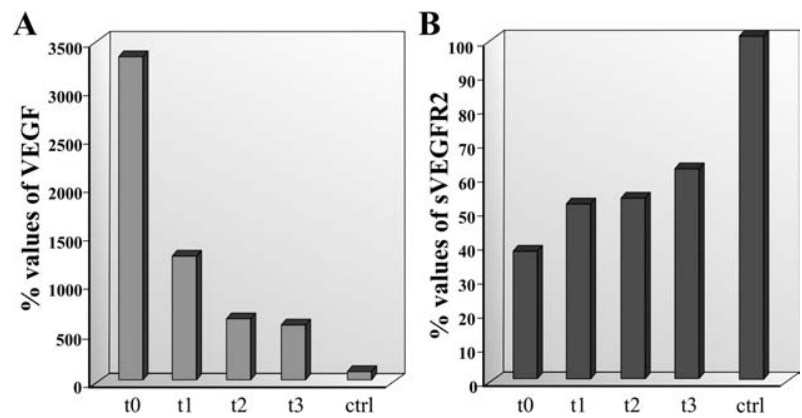

FIGURE 3. VEGF and sVEGFR2 serum level in POEMS patient and in normal controls. VEGF (A) and sVEGFR2 (B) levels were analyzed at the onset of relapse (t0) and at follow-up of 3 (t1), 6 (t2), and 9 (t3) months. An inverse correlation between VEGF and sVEGFR2 levels during the clinical course is evident. The results are from three independent assays. Bars indicate percentage values compared with the mean of 3 healthy controls.

$\mathrm{pg} / \mathrm{ml}$ ). Interestingly, the inverse correlation between VEGF and sVEGFR2 levels was maintained during the clinical course; however, both levels returned toward normal in response to therapy. These findings suggest that VEGF and sVEGFR2 levels are mutually regulated.

\section{DIscussion}

To the best of our knowledge this is the first case of POEMS presenting as relapsing AIDP, because this syndrome is generally associated with a chronic neuropathy. The rapid response to IVIg therapy, both at onset and on relapse, is in line with the general experience with AIDP, but it is unreported so far in POEMS patients. Human IgG is known to contain specific antibodies against several cytokines. ${ }^{10}$ Inflammatory cytokines, such as tumor necrosis factor- $\alpha$ (TNF- $\alpha)$, interleukin- $1 \beta$ (IL-1 $\beta$ ), and IL-6, are able to stimulate VEGF release and this, in turn, downregulates cellular VEGFR2 expression. ${ }^{2,6,11}$ On the other hand, VEGF regulates VEGFR2 density on cell surfaces by activating a process known as "shedding" which involves the cleavage and release of a soluble ectodomain from the membrane-anchored form of the receptor. ${ }^{12}$ Thus, the release of soluble VEGFR2, which naturally occurs in plasma, should decrease its availability on cells and simultaneously generate a soluble decoy that might intercept VEGF. ${ }^{6}$

The reduction of the sVEGFR2 level found in our patient might result from either downregulation of VEGFR2 gene expression, as previously suggested, ${ }^{13}$ and/or from reduced shedding of the membrane-integrated form. However, the mechanisms postulated in POEMS syndrome, such as basal lamina thickening, endothelial cell proliferation, and opening of tight junctions in endoneurial microvessels, all leading to blood-nerve barrier breakdown, ${ }^{14}$ seem to implicate activation of the VEGF-VEGFR2 pathway. 
We hypothesize, therefore, that in our case, IVIg treatment was able to prevent progression and to reverse initial nerve fiber damage through neutralization of circulating cytokines with rapid remission of neuropathic symptoms. The substantial reduction of VEGF and the increase of circulating VEGFR2, observed after IVIg and radiation therapies, probably prevented persistent endothelial activation, endoneurial edema, and evolution to a chronic neuropathy. ${ }^{14}$

The main findings of this study are that: (1) POEMS may present with an acute polyneuropathy that can be successfully treated with IVIg infusion; and (2) in POEMS, a rise in VEGF levels can be accompanied by a reduction of its soluble receptor sVEGFR2. This finding seems to correlate with the clinical course and response to therapy.

This work was supported by Grant 2005060584_005 from the Italian Ministry of University and Research (PRIN). The authors thank Graziano Bonelli for excellent technical assistance.

\section{REFERENCES}

1. Watanabe $\mathrm{O}$, Arimura K, Kitajima I, Osame M, Maruyama I. Greatly raised vascular endothelial growth factor (VEGF) in POEMS syndrome. Lancet 1996;347:702.

2. Dispenzieri A. POEMS syndrome. Blood Rev 2007;21:285-299.

3. Dyck PJ, Engelstad JN, Dispenzieri A. Vascular endothelial growth factor and POEMS. Neurology 2006;66:10-12.
4. Ruiz de Almodovar C, Lambreechts D, Mazzone M, Carmeliet P. Role and therapeutic potential of VEGF in nervous system. Physiol Rev 2009;89:607-648.

5. Olsson AK, Dimberg A, Kreuger J, Claesson-Welsh L. VEGF receptor signalling-in control of vascular function. Nat Rev Mol Cell Biol 2006;7:359-371.

6. Ebos JML, Bocci G, Man S, Thorpe PE, Hickland DJ, Zhou D, et al. A naturally occurring soluble form of vascular endothelial growth factor receptor 2 detected in mouse and human plasma. Mol Cancer Res 2004;2:315-326.

7. van Koningsveld R, Steyerberg EW, Hughes RA, Swan AV, van Doorn PA, Jacobs BC. A clinical prognostic scoring system for GuillainBarré syndrome. Lancet 2007;6:589-594.

8. George ML, Eccles SA, Tutton MG, Abulafi AM, Swift RI. Correlation of plasma and serum vascular endothelial growth factor levels with platelet count in colorectal cancer: clinical evidence of platelet scavenging? Clin Cancer Res 2000;6:3147-3152.

9. Nobile-Orazio E, Terenghi F, Giannotta C, Gallia F, Nozza A. Serum VEGF levels in POEMS syndrome and in immune-mediated neuropathies. Neurology 2009;72:1024-1026.

10. Svenson M, Hansen MB, Bendtzen K. Binding of cytokines to pharmaceutically prepared human immunoglobulin. J Clin Invest 1993; 92:2533-2539

11. Giraudo E, Primo L, Audero E, Gerber H-P, Koolwijk P, Soker S, et al. Tumor necrosis factor-alpha regulates expression of vascular endothelial growth factor receptor-2 and of its co-receptor neuropilin-1 in human vascular endothelial cells. J Biol Chem 1998;273: 22128-22135.

12. Swendeman S, Mendelson K, Weskamp G, Horiuchi K, Deutsch U, Schere P, et al. VEGF-A stimulates ADAM-17-dependent shedding of VEGFR2 and crosstalk between VEGFR2 and ERK signaling. Circ Res 2008;103:916-918.

13. Scarlato M, Previtali SC, Carpo M, Pareyson D, Briani C, Del Bo R, et al. Polyneuropathy in POEMS syndrome: role of the angiogenic factors in the pathogenesis. Brain 2005;128:1911-1920.

14. Zachary I. Neuroprotective role of vascular endothelial growth factor: signalling mechanisms, biological function, and therapeutic potential. Neurosignals 2005;14:207-221.

\title{
SEIPIN S90L MUTATION IN AN ITALIAN FAMILY WITH CMT2/dHMN AND PYRAMIDAL SIGNS
}

\author{
M. LUIGETTI, MD, ${ }^{1}$ G.M. FABRIZI, MD, ${ }^{2}$ F. MADIA, MD, PhD, ${ }^{1}$ M. FERRARINI, PhD, ${ }^{2}$ A. CONTE, MD, ${ }^{1}$ A. DELGRANDE, MD, ${ }^{1}$ \\ P.A. TONALI, MD, ${ }^{1,3}$ and M. SABATELLI, MD ${ }^{1}$ \\ ${ }^{1}$ Institute of Neurology, Catholic University of Sacred Heart, Largo F. Vito 1, 00168, Rome, Italy \\ ${ }^{2}$ Department of Neurological Sciences and Vision, University of Verona, Verona, Italy \\ ${ }^{3}$ Don Carlo Gnocchi Onlus Foundation, Italy \\ Accepted 22 March 2010
}

ABSTRACT: Heterozygous mutations in the Berardinelli-Seip congenital lipodystrophy (BSCL2) gene have been associated with different clinical phenotypes including Silver syndrome/ spastic paraplegia 17, distal hereditary motor neuropathy type $\mathrm{V}$, and Charcot-Marie-Tooth disease type 2 (CMT2) with predominant hand involvement. We studied an Italian family with a CMT2 phenotype with pyramidal signs that had subclinical sensory involvement on sural nerve biopsy. Direct sequencing analysis of the BSCL2 gene in the three affected siblings revealed an S90L mutation. This report confirms the variability of clinical phenotypes associated with a BSCL2 Ser9OLeu mutation and describes the first Italian family with this mutation.

Muscle Nerve 42: 448-451, 2010

Abbreviations: BSCL2, Berardinelli-Seip congenital lipodystrophy; CMAP, compound muscle action potential; CMT, Charcot-Marie-Tooth disease type; $\mathrm{dHMN}$, distal hereditary motor neuropathy; SNAP, sensory nerve action potential; SS, Silver syndrome

Key words: BSCL2; SEIPIN; CMT; dHMN; sural nerve; pyramidal involvement

Correspondence to: M. Sabatelli; e-mail: msabatelli@rm.unicatt.it

(c) 2010 Wiley Periodicals, Inc.

Published online 15 August 2010 in Wiley InterScience (www.interscience. wiley.com). DOI 10.1002/mus.21734
The Seipin/BSCL2 gene was originally associated with congenital generalized lipodystrophy type 2, an autosomal recessive illness clinically defined by severe lipoatrophy, insulin resistance, hypertriglyceridemia, and mental retardation. ${ }^{1}$ Heterozygous mutations of this gene have been described in autosomal dominant motor neuron diseases including Silver syndrome/spastic paraplegia 17 (SPG17) (OMIM \#270685) and distal hereditary motor neuropathy type V (dHMN-V) (OMIM \#182960). ${ }^{2}$ In the latter conditions only two mutations have been identified (N88S and S90L).

dHMN-V is characterized by marked and progressive symmetrical or asymmetrical weakness and wasting of the distal muscles of the upper limbs without sensory involvement; foot deformity and peroneal muscle weakness may also be present. ${ }^{3}$ Silver syndrome (SS) is a rare motor neuron 